\title{
Systemic lupus erythematosus presenting as fulminant lupus pneumonitis: a rare case report
}

\author{
H.K. Aggarwal', D. Jain 1', A. Mittal', A. Rao', R.K. Yadav', P. Jain ${ }^{2}$ \\ ${ }^{1}$ Department of Medicine, Pt. B.D. Sharma University of Health Sciences, Rohtak, India; \\ ${ }^{2}$ Department of Pathology, Pt. B.D. Sharma University of Health Sciences, Rohtak, India
}

\section{SUMMARY}

We report a case of 19 year-old female patient diagnosed as systemic lupus erythematosus (SLE) presented with fever and diffuse cutaneous lesions. During the hospital stay she had acute pneumonia, pleural effusion and respiratory failure, which required intensive care unit (ICU) care and mechanical ventilator support. A fulminant course of the disease, decreased values of complement levels and positive antinuclear antibodies (ANA) in pleural fluid and repeated negative sputum for acid-fast bacillus, blood cultures enabled diagnosis of fulminant lupus pneumonitis. Fulminant lupus pneumonitis is a rare but potentially life threatening complication of SLE. Management requires involvement of multiple specialties and rigorous efforts in reviving the patient.

Key words: Systemic lupus erythematosus; lupus pneumonitis; autoimmune systemic disease; intensive care unit.

Reumatismo, 2016; 68 (1): 48-52

\section{INTRODUCTION}

ystemic lupus erythematosus (SLE) is an autoimmune systemic disease, which can involve several organs such as skin, lungs, brain and heart. Pulmonary manifestations of SLE can include a wide spectrum of diseases such as pleuritis, pneumonia, pulmonary embolism, pneumothorax and pulmonary haemorrhage (1, 2 ). Only few cases of non-infectious fulminant lupus pneumonitis (LP) mimicking, atypical pneumonia have been presented in literature to date. Differential diagnosis and treatment of this condition represent a real challenge. We hereby report a case of SLE manifesting as fulminant LP in a young female patient.

\section{CASE REPORT}

A 19 year-old female presented with history of intermittent, high-grade fever associated with chills and multiple erythematous rashes all over the body for one week. She also complained of joint pain involving knee and elbow, recurrent oral ulcers and oligomenorrhea, which were persistent for one year. There was no history of tuberculosis, diabetes mellitus, decreased urine, chest discomfort, pedal edema, decreased vision, any bleeding episodes or recent drug intake.

On general examination, patient was conscious, well oriented in time, place and person with stable vitals. Pallor was present. Cyanosis, jaundice, clubbing and lymphadenopathy were absent. Jugular venous pressure was normal. There were multiple blanchable, erythematous rashes present all over the body (Figure 1). On respiratory system examination, breath sounds were decreased in right infra-mammary, infra-axillary and infra-scapular areas. On abdomen examination liver was palpable. The remaining systemic examination including cardiovascular system, was within normal limits. Laboratory investigations on the day of admission revealed that the patient had anemia with hemoglobin of $9.7 \mathrm{~g} / \mathrm{dl}$, but normal leukocyte and platelet counts. Erytrocyte sedimentation rate (ESR) was 25 $\mathrm{mm} / 1^{\text {st }} \mathrm{h}$. Renal function tests were deranged with blood urea of $82 \mathrm{mg} / \mathrm{dL}$ (1050) and serum creatinine of $1.6 \mathrm{mg} / \mathrm{dL}$ (0.7-1.3). Liver function tests were normal. 


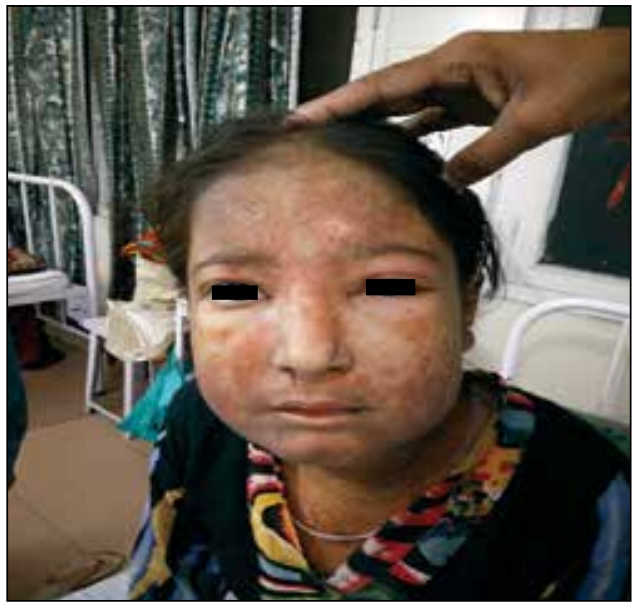

Figure 1 - Photograph of the patient at the time of presentation (with permission).

Hypoproteinemia was evident with total serum protein of $5.4 \mathrm{~g} / \mathrm{dL}$. Serum calcium, serum phosphate and fasting blood sugar level were normal. Urine complete examination revealed proteinuria. The 24 hour urine analysis showed a proteinuria of 3.2 g/day. Kidney biopsy showed grade IV lupus nephritis (Figure 2).

Blood and urine cultures were sterile. hsCRP level was $300 \mathrm{mg} / \mathrm{dL}(<10)$ and rheumatoid factor was negative. Tests for HIV, HbsAg and Anti HCV were negative. Mantoux test and sputum examination for acid fast bacillus were negative.

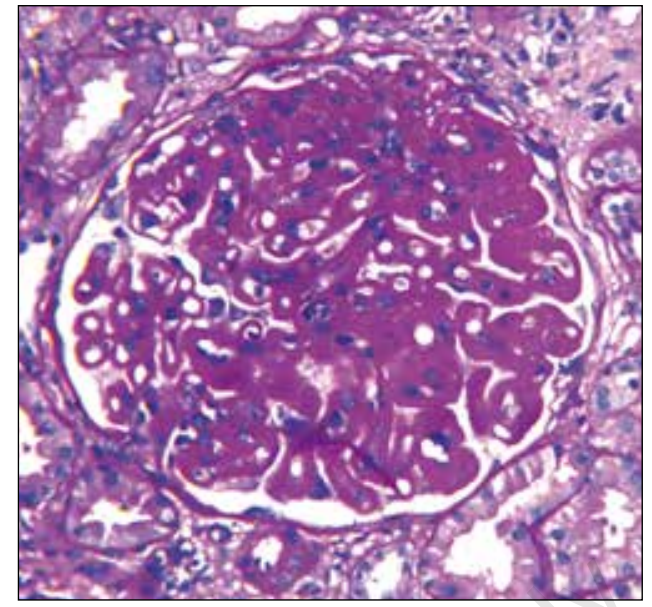

Figure 2 - Histopathology of renal biopsy specimen showing grade IV lupus nephritis.

Chest x-ray showed bilateral pleural effusion in the right lower zone and non- homogenous opacities in the right upper and mid zones (Figure 3A). High-resolution computed tomography (HRCT) of thorax showed bilateral pleural effusion, with fluid in the right major fissure. An ill-defined area of consolidation was seen in anterior segment of right upper lobe and medial segment of right middle lobe (Figure 3B). Ultrasonography of abdomen showed enlarged liver with a size of 16 centimeters. Tests for antinuclear antibodies (ANA) by immunofluorescence technique were

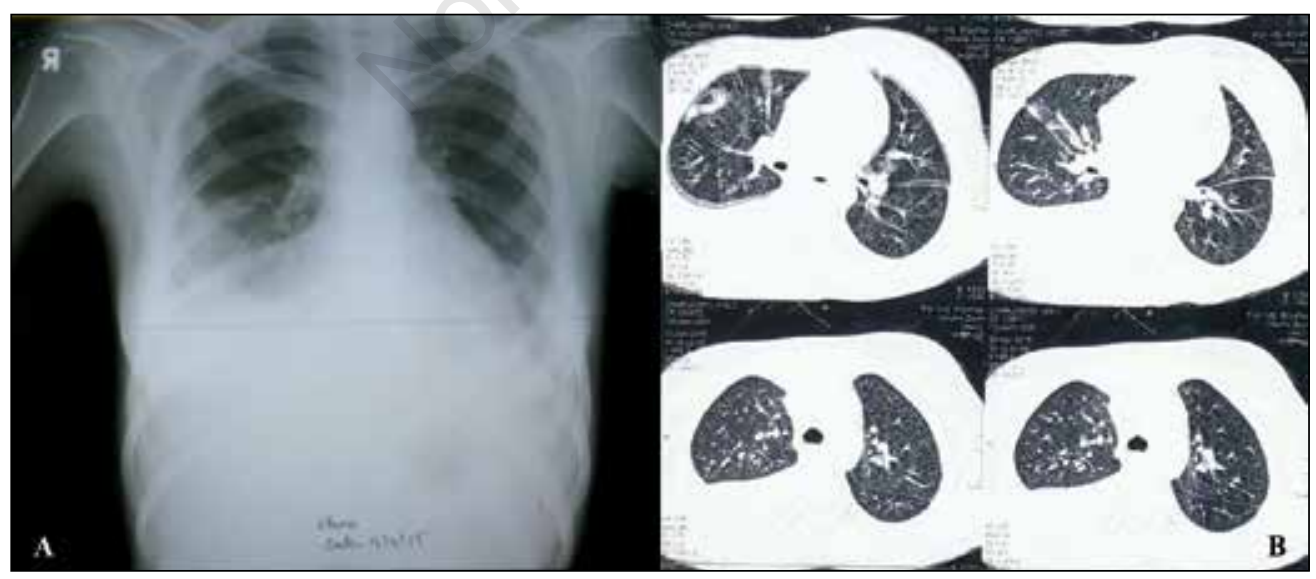

Figure 3 - A) Chest $x$-ray posterioranterior view showing bilateral pleural effusion in the right lower zone and non- homogenous opacities in the right upper and mid zones; B) High-resolution computed tomography of thorax showing bilateral pleural effusion, with fluid in the right major fissure. An ill-defined area of consolidation is seen in anterior segment of right upper lobe and medial segment of right middle lobe. 
positive with titers of $1 / 200$ and having speckled pattern; serum anti dsDNA antibodies (normal value $-<0.9$, patient's value - 1.76), anti (RO) (normal value - $<3$, patient's value - 10.72), anti (La) (normal value $-<3$, patient's value 9.95) were positive. Shirmer's test was negative and small salivary gland biopsy from buccal area was normal.

Pleural fluid analysis revealed total protein of $5.4 \mathrm{~g} / \mathrm{dL}$, total sugar of $101.1 \mathrm{mg} / \mathrm{dL}$, total leucocytes count $180 /$ cumm with predominance of lymphocytes and negative adenosine aminohydrolase (ADA). In the background of underlying SLE, the pleural fluid was sent for complement level analysis and it revealed decreased titers of $\mathrm{C} 3$ (normal value - 0.9-1.8, patient's value $0.3 \mathrm{~g} / \mathrm{dL}$ ), C4 (normal value - 0.1-0.4 g/dL, patient's value $-0.05 \mathrm{~g} / \mathrm{dL}$ ) and increased ANA titers (normal value $-<0.9$, patient's value - 1.65).

In a short span of two days the patient progressed into type 1 respiratory failure. Intensive Care Unit (ICU) care was given to the patient. She was put on mechanical ventilation on volume support mode and intravenous methylprednisolone along with empirical antibiotics. Unfortunately, patient succumbed to illness.

\section{DISCUSSION}

Fulminant LP is a rare but life threatening complication of SLE. Broadly speaking, pulmonary complications of SLE can be divided into three categories: infectious, malignant, and disease related. Although pulmonary infections typically affect the airways and/or parenchyma, complications attributable to SLE can affect all compartments of the lungs and include pleuritis (with or without effusion), interstitial lung disease (ILD), alveolar hemorrhage, shrinking lung syndrome (SLS), pulmonary hypertension $(\mathrm{PH})$, airways disease, and thromboembolic disease.

LP is a highly fatal syndrome characterized by acute onset of fever, pleuritic chest pain, and tachypnea; up to $50 \%$ mortality rate is seen with this rare condition. It is often accompanied with auscultatory crackles and hemoptysis may rarely occur. Chest imaging usually reveals bilateral opacities. Pneumonitis may be a precursor to chronic ILD in a subset of patients. In one case series, 3 of 12 patients with pneumonitis progressed to ILD, despite treatment with high-dose corticosteroids. In both LP and SLE-ILD, immune complexes, lymphocytic aggregates, and vascular pathology are common (3). There seems to be some association between (ALP) and anti-Ro/ SSa antibodies. The high frequency of anti$\mathrm{Ro} / \mathrm{SSa}$ antibodies raises the possibility of their role in the pathogenesis of ALP (4). Pleural effusions are often bilateral and small; rarely, effusions from SLE-disease activity involve more than two-thirds of the lung fields (5). It is important not only to make a diagnosis of an effusion but also to determine the underlying cause. Common causes of a pleural effusion in SLE are cardiac or renal failure (6). Consideration of underlying $\mathrm{PH}$, especially in the setting of heart failure, needs to be considered especially among patients seropositive for antiphospholipid antibodies. A thoracocentesis may be performed when there is a concern for infection, in particular for atypical infections such as tuberculosis, or malignancy. Screening pleural fluid for ANA in patients with SLE may aid in differentiating effusions related to disease activity from other etiologies. A pleural fluid ANA titer of $\geq 1 / 160$ has a sensitivity of 92\% for SLE pleuritis when compared with all other etiologies for pleural effusions and is particularly helpful when screening exudative effusions in SLE (7).

Bronchoalveolar lavage reveals histological features, which are non-specific and include alveolar wall damage and necrosis, inflammatory cell infiltration, edema, hemorrhage, and hyaline membranes. A microangiitis involving capillaries, with fibrin thrombi and infiltration with necrotic neutrophils, may be present. These histological criteria are similar to acute alveolar haemorrhage (8).

Fulminant LP tends to affect younger patients and those with recent diagnosis of SLE. In $50 \%$ of patients with SLE who develop ALP, the pulmonary complication 
is the initial presentation of lupus. Clinical presentation includes abrupt onset of fever, cough, dyspnea, pleuritic chest pain and occasionally hemoptysis. Physical examination usually reveals signs of hypoxia and bibasilar crackles. Radiographic findings include bilateral alveolar infiltrates with predominance in lower lung fields. ${ }^{3}$ Pleural effusion occurs in half of the cases. Rarely the initial chest radiograph may be normal or may show pulmonary nodules. Computed tomography (CT) scan of the chest may show diffuse ground glass opacities and areas of consolidation (9).

It is not surprising that there may be increased risk of infection in SLE for many reasons, including end-organ involvement (in particular, renal disease), high disease activity, or treatment with immunosuppressants; however, studies are conflicting about which mechanisms drive the infection risk (10). In a large prospective cohort of SLE, $25 \%$ of SLE patients developed an infection in a 5-year follow-up period; the most common site of infection was the respiratory tract and the most common organism was bacteria $(11,12)$. Although the presentation of the patients with acute fulminant pneumonitis and typical/atypical pneumonias does not differ much, the investigation and treatment modalities have a large gap. When typical/atypical pneumonias are suspected, gram staining, culture and nuclear studies have to be performed immediately to find the etiology, and an appropriate treatment has to be initiated. Pleural fluid analysis for fulminant pneumonitis plays a key role in timely diagnosis. High-dose corticosteroids including bolus intravenous methylprednisolone together with supportive measures such as oxygen and mechanical ventilation, where necessary, are still the mainstay of drug therapy for acute lupus pneumonitis, although no controlled trials have established their efficacy (13). The effusion, when present, begins to clear within days of beginning steroid therapy, though the radiographic changes may take several weeks to clear up completely. Chest tube drainage is not necessary. When clinical or radiographic changes are slow or pa- tients fail to respond to steroid therapy or relapse on steroid dose tapering, the addition of a steroid sparing agent, such as azathioprine, may be considered (14). Intravenous pulse cyclophosphamide combined with systemic corticosteroids has also been used successfully (15). In chronic, unremitting lupus pleurisy, refractory to medical therapy, pleurectomy, talc poudrage and tetracycline pleurodesis may be used. Intravenous immunoglobulin therapy is of limited value in a patient with lupus and refractory pleural effusion.

\section{CONCLUSIONS}

Diagnosis of fulminant LP is a real challenge. In the case we presented, the diagnosis has been made based on data from several negative cultures and striking history of sudden reduction of complement levels in pleural fluid. Intensive immunosuppressive treatment and, in selected cases, plasmapheresis should be introduced. A close cooperation between ICU, pulmonology and rheumatology departments is required in such a case to minimalize the risk of fatal outcomes.

Conflict of interest: the authors declare no potential conflict of interest.

\section{REFERENCES}

1. Beresford MW, Cleary AG, Sills JA, et al. Cardio-pulmonary involvement in juvenile systemic lupus erythematosus. Lupus 2005; 14: $152-8$.

2. Ciftçi E, Yalçinkaya F, Ince E, et al. Pulmonary involvement in childhood-onset systemic lupus erythematosus: a report of five cases. Rheumatology 2004; 43: 587-91.

3. Matthay RA, Schwarz MI, Petty TL, et al. Pulmonary manifestations of systemic lupus erythematosus: review of twelve cases of acute lupus pneumonitis. Medicine (Baltimore) 1975; 54: 397-409.

4. Cheema GS, Quismorio FP Jr. Interstitial lung disease in systemic lupus erythematosus. Curr Opin Pulm Med. 2000; 6: 424-9.

5. Swigris JJ, Fischer A, Gillis J, et al. Pulmonary and thrombotic manifestations of systemic lupus erythematosus. Chest 2008; 133: 271-80.

6. Badui E, Garcia-Rubi D, Robles E, et al. Cardiovascular manifestations in systemic lupus 
erythematosus. Prospective study of 100 patients. Angiology 1985; 36: 431-41.

7. Toworakul C, Kasitanon N, Sukitawut W, et al. Usefulness of pleural effusion antinuclear antibodies in the diagnosis of lupus pleuritis. Lupus 2011; 20: 1042-6.

8. Myers JL, Katzenstein AA. Microangiitis in lupus-induced pulmonary hemorrhage. Am J Clin Pathol 1986; 85: 552-6.

9. Gari AG, Telmesani A, Alwithenani R. Pulmonary manifestations of systemic lupus erythematosus. In: Almoallim H, ed. Systemic lupus erythematosus. Rijeka, Croatia: Intechopen; 2012. pp 313-36.

10. Kinder BW, Freemer MM, King TE Jr, et al. Clinical and genetic risk factors for pneumonia in systemic lupus erythematosus. Arthritis Rheum. 2007; 56: 2679-86.

11. Gladman DD, Hussain F, Ibañez D, Urowitz
MB. The nature and outcome of infection in systemic lupus erythematosus. Lupus 2002; 11: 234-9.

12. Weng CT, Liu MF, Weng MY, et al. Pneumocystis jirovecii pneumonia in systemic lupus erythematosus from southern Taiwan. J Clin Rheumatol. 2013; 19: 252-8.

13. Inoue T, Kanayama Y, Ohe A, et al. Immunopathologic studies of pneumonitis in systemic lupus erythematosus. Ann Intern Med. 1979; 91: 30-4.

14. Matthay RA, Schwartz MI, Petty TL, et al. Pulmonary manifestations of systemic lupus erythematosus: review of twelve cases of acute lupus pneumonitis. Medicine 1975; 54 : 397-409.

15. Eiser AR, Shanies HM. Treatment of lupus interstitial lung disease with intravenous cyclophosphamide. Arthritis Rheum. 1994; 37: 428-31. 